\title{
Abundances of planetary nebulae NGC 2022, NGC 6818 and IC 4191
}

\author{
S. R. Pottasch ${ }^{1}$, D. A. Beintema ${ }^{1,2}$, and W. A. Feibelman ${ }^{3}$ \\ 1 Kapteyn Astronomical Institute, PO Box 800, 9700 AV Groningen, The Netherlands \\ e-mail: pottasch@astro.rug.nl \\ 2 SRON Laboratory for Space Research, PO Box 800, 9700 AV Groningen, The Netherlands \\ 3 Laboratory for Astronomy and Solar Physics, Code 681, Goddard Space Flight Center MD, USA
}

Received 30 December 2004 / Accepted 11 March 2005

\begin{abstract}
The ISO and IUE spectra of the elliptical nebulae NGC 2022, NGC 6818 and IC 4191 are presented. These spectra are combined with the spectra in the visual wavelength region to obtain a complete, extinction corrected, spectrum. The chemical composition of the nebulae is then calculated and compared to previous determinations. A discussion is also given of the exciting stars of the nebulae, and possible evolutionary effects.
\end{abstract}

Key words. ISM: abundances - ISM: planetary nebulae: individual: NGC 2022 - ISM: planetary nebulae: individual: NGC 6818 - ISM: planetary nebulae: individual: IC 4191

\section{Introduction}

NGC 2022, NGC 6818 and IC 4191 are morphologically quite similar; the first two are usually classified as elliptical in shape (Stanghellini et al. 1993) and they are located rather far from the galactic plane $\left(11^{\circ}\right.$ and $18^{\circ}$ respectively), which is probably an indication that they have been formed from low mass stars. IC 4191 is somewhat elliptical in shape but is usually classified as "irregular". It is $5^{\circ}$ below the galactic plane. NGC 2022 has a diameter of roughly $28^{\prime \prime}$, and it has a halo which extends to about 40" diameter (Corradi et al. 2003). NGC 6818 has a diameter of about 33" (Schwarz et al. 1992) with the brightest parts toward the central regions. IC 4191 has a diameter of about $12^{\prime \prime}$ and it does not have a halo. It has an extension to the northeast, which is probably the reason that it is classified as "irregular".

The purpose of this paper is to study the element abundances in these nebulae with the help of the mid-infrared ISO spectra. Abundances in all three nebulae have been studied for many years already, but the inclusion of the ISO spectra permits a more accurate determination. The reasons for this have been discussed in earlier papers (e.g. see Pottasch \& Beintema 1999; Pottasch et al. 2000, 2001; Bernard Salas et al. 2001), and can be summarized as follows.

The most important advantage is that the infrared lines originate from very low energy levels and thus give an abundance

^ Based on observations with ISO, an ESA project with instruments funded by ESA Member States (especially the PI countries: France, Germany, The Netherlands and the UK) and with the participation of ISAS and NASA. which is not sensitive to the temperature in the nebula, nor to possible temperature fluctuations. Furthermore, when a line originating from a high-lying energy level in the same ion is observed, it is possible to determine an effective temperature at which the lines in that particular ion are formed. When the effective temperature for many ions can be determined, it is possible to make a plot of effective temperature against ionization potential, which can be used to determine the effective temperature for ions for which only lines originating from a high energy level are observed. Use of an effective electron temperature takes into account the fact that ions are formed in different regions of the nebula. At the same time possible temperature fluctuations are taken into account.

Use of the ISO spectra have further advantages. One of them is that the number of observed ions used in the abundance analysis is approximately doubled, which removes the need for using large "Ionization Correction Factors", thus substantially lowering the uncertainty in the abundance. A further advantage is that the extinction in the infrared is almost negligible, eliminating the need to include sometimes large correction factors.

These advantages lead to different abundances for some of the elements when compared with earlier determinations. These can be a factor of two different from earlier determinations and occasionally they differ by an order of magnitude. But sometimes they agree quite well with earlier work. For these three nebulae comparisons are shown in this paper.

The ISO diaphragm is not able to measure the entire nebula of these three in the shortward wavelength region, covering the spectral region from $2.4 \mu \mathrm{m}$ to about $30 \mu \mathrm{m}$. This is because it measures with a diaphragm of $14^{\prime \prime} \times 20^{\prime \prime}$ for wavelengths 
below $12 \mu \mathrm{m}$, and only a slightly larger diaphragm $\left(14^{\prime \prime} \times 27^{\prime \prime}\right)$ between $12 \mu \mathrm{m}$ and $27 \mu \mathrm{m}$. Above $27 \mu \mathrm{m}$ the diaphragm is $20^{\prime \prime} \times 33^{\prime \prime}$ which is sufficiently large to measure the entire nebula. The ISO LWS spectra (which cover a spectral region from $45 \mu \mathrm{m}$ until almost $200 \mu \mathrm{m}$ ), also include the entire nebula, since these spectra are taken with a diaphragm with a diameter of about $80^{\prime \prime}$.

This paper is structured as follows. First the ISO spectrum of the three nebulae is presented and discussed (in Sect. 2), together with the $6 \mathrm{~cm}$ radio continuum flux density. Section 3 discusses the visible spectrum of these nebulae and also presents the ultraviolet (IUE) spectrum, except for IC 4191 which was not measured by the IUE. This is followed by a discussion of the nebular electron temperature and density and the chemical composition of the three nebulae (Sect. 4). A comparison of the resultant abundances with those made earlier is given in Sect. 5, which also includes a discussion of the errors and the Ionization Correction Factor. In this section the relation between nebular carbon abundances determined from collisionally excited lines and from recombination lines is also studied. In Sect. 6 the central stars are considered in more detail. Finally Sect. 7 gives a general discussion.

\section{The ISO spectra}

\subsection{ISO observations of NGC 2022}

The ISO SWS observations were made with the SWSO2 observing template which observes selected lines from $2.5 \mu \mathrm{m}$ to $45 \mu \mathrm{m}$ (TDT 69702701 and 69201703). The SWS01 macro, which has not been used for these nebulae, measures the entire spectrum. The SWS02 macro measures only the neighborhood of the lines assigned to it and has a much longer integration time on these lines. But some lines may be missed. The longwave observations were made with an LWS02 observation (TDT 69201604) which measures selected lines in the range from $45 \mu \mathrm{m}$ to $190 \mu \mathrm{m}$. The two SWS02 measurements were made at slightly different positions: the measurement closest to the center of the nebula was centered at $\mathrm{RA}(2000) 05^{\mathrm{h}} 42^{\mathrm{m}} 06.13^{\mathrm{s}}$ and $\operatorname{Dec}(2000)+9^{\circ} 05^{\prime} 10.1^{\prime \prime}$. The other measurement was made about $9^{\prime \prime}$ away and gives somewhat lower intensities. Since the Brackett $\alpha$ intensity can be directly related to the $\mathrm{H} \beta$ intensity theoretically, the ratio of line intensity to $\mathrm{H} \beta$ can be calculated. This ratio is taken from Hummer \& Storey (1987) at the electron temperature given in Sect. 4. The intensity of the lines found in the better centered spectrum is shown in Col. 3 of Table 1; the ratio of the line intensity to $\mathrm{H} \beta$ is given in Col. 4 . The uncertainty of the stronger lines is less than $10 \%$, while that of the weaker lines could be as large as $30 \%$. The LWS measurements (TDT 86801205) were made at essentially the same position and measure the entire nebula. The $\mathrm{H} \beta$ intensity used for these lines is the entire $\mathrm{H} \beta$ intensity as derived from the radio measurements (see below).

\subsection{ISO spectrum of NGC 6818}

The ISO SWS observations were made only with the SWS02 observing template whose advantages and disadvantages have
Table 1. ISO spectrum of NGC 2022. The measured lines are given in Col. 3. The last column gives the ratio of the line intensity to $\mathrm{H} \beta(=100)$.

\begin{tabular}{|c|c|c|c|}
\hline Ident. & $\lambda(\mu \mathrm{m})$ & Intensity & $I / \mathrm{H} \beta$ \\
\hline H I 5-4 & 4.053 & 8.83 & \\
\hline [Mg IV] & 4.487 & 38.6 & 29.3 \\
\hline$[\mathrm{Mg} \mathrm{v}]$ & 5.611 & 23.8 & 18.1 \\
\hline$[\mathrm{Arv}]$ & 7.903 & 19.0 & 6.35 \\
\hline [Ar III] & 8.993 & 15.2 & 11.6 \\
\hline [S IV] & 10.507 & 210. & 160. \\
\hline [Ne II] & 12.815 & 13.6 & 10.3 \\
\hline [Arv] & 13.103 & 19.0 & 14.4 \\
\hline$[\mathrm{Ne} \mathrm{V}]$ & 14.323 & 176. & 134.0 \\
\hline [Ne III] & 15.556 & 74.8 & 56.8 \\
\hline [S III] & 18.715 & 22.3 & 16.9 \\
\hline [Ne III] & 36.021 & 15.6 & 8.72 \\
\hline [O III] & 51.8 & 351. & 185 \\
\hline [N III] & 57.347 & 60.2 & 33.6 \\
\hline [O III] & 88.398 & 112. & 59.2 \\
\hline
\end{tabular}

Intensity measured in units of $10^{-13} \mathrm{erg} \mathrm{cm}^{-2} \mathrm{~s}^{-1}$.

been discussed in the previous section. The longwave observations were also made with an LWS02 template, but here all important lines were measured. As in NGC 2022 the nebula is somewhat larger than the observing diaphragm for SWS measurements so that the same procedure was used. The observed intensity of the lines found in the spectrum are shown in Col. 3 of Table 2. In Col. 4 the ratio of the line intensity to $\mathrm{H} \beta(\mathrm{I} / \mathrm{H} \beta)$ as seen in the diaphragm is given. For the lines with wavelengths below $30 \mu \mathrm{m}$ the $\mathrm{H} \beta$ is found from the observed Brackett $\alpha$ line using the theoretical ratio of the two lines given by Hummer \& Storey 1987 for a temperature of $T=13000 \mathrm{~K}$ (see below). Above $30 \mu \mathrm{m}$ the diaphragm used are large enough so that the entire nebula is seen and the $\mathrm{H} \beta$ is found from the $6 \mathrm{~cm}$ radio flux density (see below). The uncertainty of the stronger lines is less than $10 \%$, increasing for the weaker lines to $30 \%$. The SWS02 measurement (TDT 34301004) was centered at RA(2000) $19^{\mathrm{h}} 43^{\mathrm{m}} 57.73^{\mathrm{s}}$ and Dec(2000) $-14^{\circ} 09^{\prime} 11.7^{\prime \prime}$, and the LWS02 measurement (TDT 34301005) was at essentially the same position. Liu et al. (2001) did not report LWS measurements of this nebula.

\subsection{ISO spectrum of IC 4191}

IC 4191 is a compact nebula with a diameter of about 7" so that the entire nebula normally would be measured by the ISO diaphragm. Unfortunately the position measured was about 8 " from the center of the nebula. The SWS02 measurement (TDT 48800165) was centered at RA(2000) $13^{\mathrm{h}} 08^{\mathrm{m}} 48.55^{\mathrm{s}}$ and $\operatorname{Dec}(2000)-67^{\circ} 38^{\prime} 32.6^{\prime \prime}$ while the center of the nebula is $\operatorname{RA}(2000) 13^{\mathrm{h}} 08^{\mathrm{m}} 47.61^{\mathrm{s}}$ and $\operatorname{Dec}(2000)-67^{\circ} 38^{\prime} 37.7^{\prime \prime}$. Thus a correction was necessary for all lines below $30 \mu \mathrm{m}$. This correction was found in the same way as was done for the two other nebulae, by comparing the $\mathrm{H} \beta$ flux predicted by 
Table 2. ISO observations of NGC 6818. (The measured intensities are in units of $10^{-12} \mathrm{erg} \mathrm{cm}^{-2} \mathrm{~s}^{-1}$.)

\begin{tabular}{|c|c|c|c|}
\hline Ident. & $\lambda(\mu \mathrm{m})$ & Intensity & $I / \mathrm{H} \beta$ \\
\hline He II 9-7 & 2.826 & 0.416 & \\
\hline Н I 5-4 & 4.052 & 2.78 & \\
\hline [Mg IV] & 4.486 & 8.77 & 0.214 \\
\hline Н I 8-6 & 7.460 & 1.28 & \\
\hline$[\mathrm{Arv}]$ & 7.902 & 1.27 & 0.0309 \\
\hline [Ar III] & 8.991 & 6.32 & 0.154 \\
\hline [S IV] & 10.510 & 54.7 & 1.33 \\
\hline [Ne II] & 12.813 & 1.10 & 0.0268 \\
\hline [Arv] & 13.101 & 2.05 & 0.0500 \\
\hline$[\mathrm{Mg} \mathrm{v}]$ & 13.520 & 0.409 & 0.00996 \\
\hline$[\mathrm{Ne} \mathrm{v}]$ & 14.321 & 41.0 & 0.999 \\
\hline [Ne III] & 15.554 & 53.7 & 1.31 \\
\hline [S III] & 18.712 & 15.4 & 0.375 \\
\hline [Ar III] & 21.830 & 0.951 & 0.0232 \\
\hline$[\mathrm{Ne} \mathrm{v}]$ & 24.317 & 53.9 & 1.31 \\
\hline [O IV] & 25.889 & 346. & 8.43 \\
\hline [S III] & 33.479 & 14.5 & 0.195 \\
\hline [Ne III] & 36.012 & 7.77 & 0.105 \\
\hline [O III] & 51.816 & 124. & 1.67 \\
\hline [N III] & 57.332 & 24.8 & 0.347 \\
\hline [O III] & 88.356 & 52.5 & 0.707 \\
\hline
\end{tabular}

converting from the observed Brackett $\alpha$ and $\beta$ intensities using the theoretical ratios of Hummer \& Storey at a temperature of $10000 \mathrm{~K}$. This correction amounts to about a factor of 1.5 . The observed line fluxes are shown in Col. 3 of Table 3 and the value of $I / \mathrm{H} \beta$ in Col. 4. No correction was necessary for lines with wavelengths above $30 \mu \mathrm{m}$. The LWS02 measurement (TDT 48800166) was made at essentially the same position as the SWS02 measurement. The measurements given are taken from Liu et al. (2001).

\section{Extinction}

There are several methods for obtaining the extinction: (1) comparison of radio emission with $\mathrm{H} \beta$ flux; (2) comparison of observed and theoretical Balmer decrement; (3) dip at $\lambda 2200 \AA$; (4) photometry of the exciting star. Only the first two methods are will be discussed here. First we will discuss the radio emission and the $\mathrm{H} \beta$ flux.

\subsection{The $6 \mathrm{~cm}$ radio emission}

\subsubsection{NGC 2022}

The $6 \mathrm{~cm}$ flux density has been measured in the PMN survey (Griffith et al. 1994). They find $73 \mathrm{mJy}$ with a fixed width fit. The nebula has also been measured by Milne \& Aller (1982) who find $96 \mathrm{mJy}$ at $2 \mathrm{~cm}$, which, if optically thin, would imply $108 \mathrm{mJy}$ at $6 \mathrm{~cm}$. Becker et al. (1991) give $80 \mathrm{mJy}$ and, using the same data, Gregory \& Condon (1991) find
Table 3. ISO spectrum of IC 4191. Column 3 gives our measured values and Col. 4 the ratios of the corrected intensities to $\mathrm{H} \beta$. (All intensities are in units of $10^{-12} \mathrm{erg} \mathrm{cm}^{-2} \mathrm{~s}^{-1}$.)

\begin{tabular}{lrcc}
\hline \hline Ident. & $\lambda(\mu \mathrm{m})$ & Intensity & $I / \mathrm{H} \beta$ \\
\hline H I 6-4 & 2.626 & 1.42 & \\
H I 5-4 & 4.052 & 2.47 & \\
{$[\mathrm{Mg}$ IV] } & 4.486 & 2.69 & 0.081 \\
{$[$ Ar III] } & 8.991 & 9.28 & 0.294 \\
{$[\mathrm{~S} \mathrm{IV}]$} & 10.510 & 63.8 & 2.02 \\
{$[\mathrm{Ne}$ III] } & 15.554 & 140. & 4.19 \\
{$[\mathrm{~S} \mathrm{III}]$} & 18.712 & 14.3 & 0.433 \\
{$[\mathrm{Ar}$ III] } & 21.831 & 0.628 & 0.0188 \\
{$[\mathrm{O}$ IV] } & 25.889 & 48.3 & 1.45 \\
{$[\mathrm{~S} \mathrm{III}]$} & 33.478 & 4.40 & 0.086 \\
{$[\mathrm{Ne}$ III] } & 36.011 & 11.1 & 0.217 \\
{$[\mathrm{O}$ III] } & 52. & 48. & 0.94 \\
{$[\mathrm{~N} \mathrm{III]}]$} & 57. & 5. & 0.098 \\
{$[\mathrm{O}$ III] } & 88. & 13.3 & 0.26 \\
\hline
\end{tabular}

$84 \mathrm{mJy}$. We shall use $85 \mathrm{mJy}$ in the present analysis giving much weight to the last reference. This implies an $\mathrm{H} \beta$ flux of $1.79 \times$ $10^{-11} \mathrm{erg} \mathrm{cm}^{-2} \mathrm{~s}^{-1}$.

\subsubsection{NGC 6818}

This nebula has also been measured in the PMN survey by Griffith et al. (1994), who find 296 mJy with a fixed width fit, and $299 \mathrm{mJy}$ with a general width fit. The nebula has also been measured by Milne \& Aller (1982) who find $270 \mathrm{mJy}$ at $2 \mathrm{~cm}$ which, if optically thin, would imply $304 \mathrm{mJy}$ at $6 \mathrm{~cm}$. We shall use an average value of $298 \mathrm{mJy}$, which implies an $\mathrm{H} \beta$ flux of $7.43 \times 10^{-11} \mathrm{erg} \mathrm{cm}^{-2} \mathrm{~s}^{-1}$.

\subsubsection{IC 4191}

The PMN survey (Griffith et al. 1994) finds $171 \mathrm{mJy}$ with a fixed width fit, and $190 \mathrm{mJy}$ with a general width fit. The nebula has also been measured by Milne \& Aller (1982) who find $152 \mathrm{mJy}$ at $2 \mathrm{~cm}$ which, if optically thin, would imply $173 \mathrm{mJy}$ at $6 \mathrm{~cm}$. Cahn et al. (1992) give $170 \mathrm{mJy}$. We shall use $171 \mathrm{mJy}$, which implies an $\mathrm{H} \beta$ flux of $5.25 \times 10^{-11} \mathrm{erg} \mathrm{cm}^{-2} \mathrm{~s}^{-1}$.

\subsection{The $H \beta$ flux}

The measured $\mathrm{H} \beta$ fluxes summarized by Cahn et al. (1992) will be used. For NGC $2022 \mathrm{H} \beta=7.45 \times 10^{-12} \mathrm{erg} \mathrm{cm}^{-2} \mathrm{~s}^{-1}$, for NGC $6818 \mathrm{H} \beta=3.04 \times 10^{-11} \mathrm{erg} \mathrm{cm}^{-2} \mathrm{~s}^{-1}$ and for IC 4191 $\mathrm{H} \beta=1.02 \times 10^{-11} \mathrm{erg} \mathrm{cm}^{-2} \mathrm{~s}^{-1}$.

\subsection{The value of the extinction $E_{B-V}$}

Using the above values of the $6 \mathrm{~cm}$ radio flux densities and values of electron temperature and helium abundance which will presently be discussed, we obtain the intrinsic flux of $\mathrm{H} \beta$. 
For NGC 2022, NGC 6818 and IC 4191 the $\mathrm{H} \beta$ flux in units of $10^{-11} \mathrm{erg} \mathrm{cm}^{-2} \mathrm{~s}^{-1}$ is respectively $1.79,7.43$ and 5.25 . Combining this with the measured value of $\mathrm{H} \beta$ gives a value of extinction $C=0.38$ or $E_{B-V}=0.26$ for NGC 2022, $C=0.35$ or $E_{B-V}=0.24$ for NGC 6818 , and $C=0.70$ or $E_{B-V}=0.48$ for IC 4191. In these calculations, as well as in the rest of the paper, the extinction curve given by Fluks et al. (1994) has been used.

\subsubsection{Balmer decrement}

The extinction determined from the Balmer decrement differs somewhat according to the author. For NGC 2022 Tsamis et al. (2003) give $C=0.38$, Aller \& Czyzak (1980) give $C=0.37$ and Kwitter et al. (2003) find $C=0.21$. For NGC 6818 Tsamis et al. (2003) give $C=0.47$, Hyung et al. (1999) finds $C=0.3$ and Aller \& Czyzak (1979) give $C=0.25$. For IC 4191 Tsamis et al. (2003) give $C=0.48$.

Sometimes the agreement is reasonable, but sometimes rather large differences are found. These differences could only be real if the extinction were all local to the nebulae and varied within the nebulae and the individual authors measured at different positions in the nebulae. We regard this as unlikely; the most likely origin of these differences are errors of measurement. This is because there is no indication of extinction within the nebulae, and it is unlikely that interstellar extinction varies by such large amounts on spatial scales of a few arcsec. Besides this, the average values are very similar to the values found from the $6 \mathrm{~cm} / \mathrm{H} \beta$ method. This will have consequences in the next section where the visual spectrum is discussed.

\subsubsection{Extinction summary}

The extinction for the different wavelength regions has been corrected as follows:

1) The intrinsic value of $\mathrm{H} \beta$ used is that derived from the $6 \mathrm{~cm}$ radio flux density and is given in Sect. 3.3.

2) The infrared spectrum as observed at wavelengths greater than $30 \mu \mathrm{m}$ is assumed to come from the entire nebula. The value of extinction is quite small in this spectral region. For SWS measurements below this wavelength the ratio of the line intensity to $\mathrm{H} \beta$ is found using the Brackett $\alpha$ line. No extinction corrections are used.

3) Different observers of the visual spectrum find different extinctions from the Balmer decrement. It is assumed that the extinction to a given nebula is, in reality independent of the observer and is due to incorrect compensation of wavelength sensitivity differences by the individual observer. We assume here that for an individual observer the error in sensitivity is compensated by the error in the derived extinction. This means that the corrected spectrum relative to $\mathrm{H} \beta$ given by each individual observer will be used. For a given nebula the individual observations will be averaged. This should be approximately correct, at least over the wavelength range for which the extinction is derived, which is usually the Balmer decrement, but may be the Paschen-Balmer ratio.
4) The (IUE) ultraviolet spectrum is related to the visible spectrum by assuming a theoretical He II $\lambda 1640 / \lambda 4686$ ratio (Hummer \& Storey 1987). This corrects both for the extinction and for the somewhat smaller diaphragm of the IUE. The further correction within the ultraviolet is made using the extinction given in Sect. 3.3 and the intrinsic extinction curve of Fluks et al. (1994).

\subsection{The visual spectrum}

\subsubsection{NGC 2022}

The visual spectrum has been measured by several authors. The highest resolution spectrum is by Tsamis et al. (2003), but reliable spectra have also been reported by Aller \& Czyzak (1979) and Kwitter et al. (2003). The measurements of Tsamis et al. integrate the entire nebula, the other two cover a reasonably large area of the nebula. The measurements of Tsamis et al. are preferred both because of the higher spectral resolution used and the fact that they average the entire nebula. The results are shown in Table 4 where the Cols. 3-5 give the intensities measured by the various authors relative to $\mathrm{H} \beta=100$ for those lines which are of interest. As discussed above these lines have been corrected by the individual authors for extinction. In the last column the total intensities are given, normalized to the total flux: $\mathrm{H} \beta=1.79 \times 10^{-11} \mathrm{erg} \mathrm{cm}^{-2} \mathrm{~s}^{-1}$. An average value of the individual measurements, is used, strongly weighted to the measurements of Tsamis et al. (2003).

\subsubsection{NGC 6818}

Similarly, the visible spectrum of NGC 6818 has been measured by several authors. Their results are shown in Table 5, which are corrected for reddening by each author. Again, the measurements of Tsamis et al. (2003) are averaged over the entire nebula, the other measurements refer to smaller regions of the nebula. The last column of the table gives the total emission of the entire nebula, where the average gives more weight to the high resolution measurements, especially for the weaker lines. The [Ne v] $23425 \AA$ line intensity was taken from Rowlands et al. (1993) who had a diaphragm large enough to measure the entire nebula.

\subsubsection{IC 4191}

The visual spectrum of IC 4191 has not been extensively measured. The best measurements are those of Tsamis et al. (2003), which have been supplemented by the line ratios given by Wang et al. (2004). These line intensities are averaged over the nebula and are shown in Table 6 . The last column of the table gives the total intensity of the nebula.

\subsection{The IUE ultraviolet spectrum}

\subsubsection{NGC 2022}

Twenty IUE observations of this nebula exist, 19 are low resolution spectra and one is a high resolution shortwave spectrum. 
Table 4. Visual spectrum of NGC 2022 (total intensity in units of $10^{-12} \mathrm{erg} \mathrm{cm}^{-2} \mathrm{~s}^{-1}$ ).

\begin{tabular}{|c|c|c|c|c|c|}
\hline \multirow{2}{*}{$\begin{array}{c}\lambda \\
(\AA)\end{array}$} & \multirow[t]{2}{*}{ Ion } & \multicolumn{3}{|c|}{ Intensities } & \multirow{2}{*}{$\begin{array}{l}\text { Total } \\
\text { Intens. }\end{array}$} \\
\hline & & (1) & $(2)$ & (3) & \\
\hline 3726 & [O II $]$ & $14.1^{*}$ & 2.70 & $7.8^{*}$ & 0.96 \\
\hline 3729 & [O II] & & 2.30 & & 0.82 \\
\hline 3869 & [Ne III] & 56.5 & 41.9 & 51.7 & 9.49 \\
\hline 4102 & $\mathrm{H} \delta$ & 28.0 & 25.8 & 22.9 & 4.74 \\
\hline 4267 & C II & 0.82 & 0.89 & 0.9 & 0.15 \\
\hline 4340 & $\mathrm{H} \gamma$ & 51.1 & 45.9 & 44.9 & 8.95 \\
\hline 4363 & [O III] & 14.1 & 11.8 & 11.1 & 2.33 \\
\hline 4686 & He II & 112. & 107. & 88.8 & 18.2 \\
\hline 4711 & Ar IV & 14.3 & 13.9 & 11.4 & 2.51 \\
\hline 4724 & $\mathrm{Ne} I V$ & 1.07 & & & 0.19 \\
\hline 4726 & $\mathrm{Ne}$ IV & 0.865 & & & 0.155 \\
\hline 4740 & Ar IV & 12.4 & 11.0 & 9.0 & 2.15 \\
\hline 4861 & $\mathrm{H} \beta$ & 100 & 100 & 100 & 17.9 \\
\hline 5007 & [O III] & 735. & 675. & 640. & 130. \\
\hline 5518 & [Cl III] & 0.429 & 0.66 & 0.4 & 0.077 \\
\hline 5538 & [Cl III] & 0.358 & & & 0.064 \\
\hline 5876 & He I & 1.94 & 1.90 & 1.6 & 0.34 \\
\hline 6102 & K IV & 0.658 & 0.48 & 0.5 & 0.11 \\
\hline 6312 & [S III] & 1.88 & 1.7 & 1.7 & 0.328 \\
\hline 6563 & $\mathrm{H} \alpha$ & 300. & 286. & 282. & 51.9 \\
\hline 6584 & [N II] & 2.28 & 2.1 & 1.9 & 0.40 \\
\hline 6717 & [S II] & 0.655 & 0.46 & 0.7 & 0.116 \\
\hline 6731 & [S II] & 0.731 & 0.62 & 0.7 & 0.130 \\
\hline 7005 & [Arv] & 3.64 & 2.5 & 2.6 & 0.61 \\
\hline 7136 & [Ar III] & 9.51 & & 7.0 & 1.65 \\
\hline 7237 & [Ar IV] & 0.559 & & 0.3 & 0.068 \\
\hline 7320 & [O II] & 0.347 & 0.38 & & 0.062 \\
\hline 7330 & [O II] & 0.359 & 0.48 & & 0.068 \\
\hline 8045 & [Cl IV] & & & 1.3 & 0.23 \\
\hline 9531 & [S III] & & & 26.9: & 4.8: \\
\hline
\end{tabular}

(1) Tsamis et al. (2003); (2) Aller \& Czyzak (1979); (3) Kwitter et al. (2003).

(:) indicates uncertain values.

* This is a blend of $\lambda 3726$ and $\lambda 3729$.

We have used the large aperature $\left(10^{\prime \prime} \times 23^{\prime \prime}\right)$ measurements centered at the position of the central star which includes the major part of the nebula. For the shortwavelength spectrum we have used SWP 08676 and the longwavelength spectrum used was LWR 07428.

The extinction correction, as already explained above, was made by assuming a theoretical ratio for the He II line ratio $\lambda 1640 / \lambda 4681 \AA$ at $T=15000 \mathrm{~K}$ and an $n_{\mathrm{e}}$ of $10^{-3}$. The further extinction correction was made relative to $\lambda 1640 \AA$ using $E_{B-V}=0.26$. Correcting in this way automatically includes a diaphragm correction. The results are shown in the last column of Table 7 .
Table 5. Visual spectrum of NGC 6818 (total intensity in units of $10^{-12} \mathrm{erg} \mathrm{cm}^{-2} \mathrm{~s}^{-1}$ ).

\begin{tabular}{|c|c|c|c|c|c|}
\hline \multirow{2}{*}{$\begin{array}{c}\lambda \\
(\AA)\end{array}$} & \multirow[t]{2}{*}{ Ion } & \multicolumn{3}{|c|}{ Intensities } & \multirow{2}{*}{$\begin{array}{l}\text { Total } \\
\text { Intens. }\end{array}$} \\
\hline & & (1) & (2) & (3) & \\
\hline 3425 & {$[\mathrm{Ne} \mathrm{V}]$} & 50.0 & $83 .:$ & & $24.1^{*}$ \\
\hline 3726 & [O II] & 44.5 & 32. & 21.1 & 26.1 \\
\hline 3729 & [O II $]$ & 30.1 & 20. & 21.8 & 17.8 \\
\hline 3869 & [Ne III] & 142. & 110. & 111. & 96.6 \\
\hline 4102 & $\mathrm{H} \delta$ & 25.5 & 25.8 & 25.1 & 18.9 \\
\hline 4267 & $\mathrm{C}$ II & 0.449 & 0.44 & 0.557 & 0.34 \\
\hline 4340 & $\mathrm{H} \gamma$ & 46.8 & 46.9 & 45.3 & 34.7 \\
\hline 4363 & [O III] & 23.2 & 22.9 & 23.4 & 17.2 \\
\hline 4686 & He II & 61.1 & 71. & 70.4 & 48.0 \\
\hline 4861 & $\mathrm{H} \beta$ & 100 & 100 & 100 & 74.3 \\
\hline 5007 & [O III] & 1540. & 1740. & 2511. & 1190. \\
\hline 5518 & {$[\mathrm{Cl} \mathrm{III}]$} & 0.828 & 0.68 & 0.877 & 0.62 \\
\hline 5538 & [Cl III] & 0.867 & 0.73 & 0.807 & 0.59 \\
\hline 5755 & [N II] & 1.23 & 0.80 & 1.032 & 0.89 \\
\hline 5876 & He I & 7.46 & 5.78 & 7.02 & 5.4 \\
\hline 6101 & [K IV] & 0.350 & 0.408 & 0.60 & 0.30 \\
\hline 6312 & [S III] & 3.30 & 2.76 & 3.67 & 2.45 \\
\hline 6563 & $\mathrm{H} \alpha$ & 274.6. & 286. & 282. & 205. \\
\hline 6584 & [N II $]$ & 62.5 & 25.5 & 73.7 & 46.4 \\
\hline 6717 & [S II] & 5.30 & 2.96 & 6.64 & 3.94 \\
\hline 6731 & [S II] & 7.07 & 3.72 & 7.83 & 5.31 \\
\hline 7005 & [Ar IV] & 1.36 & 1.78 & 2.68 & 1.33 \\
\hline 7136 & [Ar III] & 18.2 & 15.1 & 28.2 & 15.5 \\
\hline 7170 & [Ar IV] & 0.26 & 0.53 & 0.497 & 0.30 \\
\hline 7320 & [O II $]$ & 2.17 & & 2.51 & 1.64 \\
\hline 7330 & [O II $]$ & 1.81 & & 2.36 & 1.41 \\
\hline 8045 & [Cl IV] & & & 1.80 & 1.3 \\
\hline 9531 & [S III] & & & 83.4 & 61.9 \\
\hline
\end{tabular}

(1) Tsamis et al. (2003); (2) Aller \& Czyzak (1979); (3) Hyung et al. (1999).

The (:) indicates uncertain values.

* The [Ne v] $\lambda 3425 \AA$ A intensity was taken from Rowlands et al. (1993).

\subsubsection{NGC 6818}

Both low and high IUE spectra have been studied by Hyung et al. (1999). We have used their results on both the low resolution shortwave spectrum SWP 01705 and the longwave spectrum LWR 01608. We have also consulted the high resolution SWP 04884 and LWR 04211 spectra to measure some weak lines and line ratios which were not resolvable on the low resolution spectra. From these spectra we found a C III $\lambda 1907 / \lambda 1909$ ratio of 1.415 and a Ne IV $\lambda 2422 / \lambda 2425$ ratio of 1.013. The low resolution intensities are shown in Table 8, where Col. 3 gives the observed intensity, Col. 4 gives the intensity corrected for extinction, and the last column give the intensity relative to $\mathrm{H} \beta=100$ normalized to the He II line ratio $\lambda 1640 / \lambda 4686 \AA=6.81$. This corrects for the diaphragm 
Table 6. Visual spectrum of IC 4191 (total intensity in units of $10^{-12} \mathrm{erg} \mathrm{cm}^{-2} \mathrm{~s}^{-1}$ ).

\begin{tabular}{|c|c|c|c|}
\hline $\begin{array}{c}\lambda \\
(\AA)\end{array}$ & Ion & $\begin{array}{c}\text { Intensity } \\
\text { (1) }\end{array}$ & $\begin{array}{c}\text { Total } \\
\text { Intensity }\end{array}$ \\
\hline 3727 & {$[\mathrm{O} \mathrm{II}]$} & 59.1 & 31.1 \\
\hline 3869 & [Ne III] & 138. & 72.6 \\
\hline 4102 & $\mathrm{H} \delta$ & 24.5 & 12.9 \\
\hline 4267 & C II & 0.546 & 0.287 \\
\hline 4340 & $\mathrm{H} \gamma$ & 46.8 & 24.6 \\
\hline 4363 & [O III] & 10.1 & 5.31 \\
\hline 4686 & He II & 13.2 & 6.94 \\
\hline 4711 & Ar IV & 2.92 & 1.55 \\
\hline 4724 & $\mathrm{Ne}$ IV & 0.17 & 0.089 \\
\hline 4725 & $\mathrm{Ne}$ IV & 0.125 & 0.066 \\
\hline 4740 & Ar IV & 4.97 & 2.6 \\
\hline 4861 & $\mathrm{H} \beta$ & 100 & 52.5. \\
\hline 5007 & [O III] & 1500. & 789. \\
\hline 5518 & [Cl III] & 0.446 & 0.234 \\
\hline 5538 & [Cl III $]$ & 0.857 & 0.433 \\
\hline 5755 & [N II] & 2.32 & 1.22 \\
\hline 5876 & He I & 17.2 & 9.03 \\
\hline 6101 & [K IV] & 0.264 & 0.14 \\
\hline 6312 & [S III] & 2.59 & 1.36 \\
\hline 6563 & $\mathrm{H} \alpha$ & 275. & 144. \\
\hline 6584 & [N II] & 82.2 & 43.2 \\
\hline 6717 & [S II] & 4.95 & 2.61 \\
\hline 6731 & [S II] & 9.29 & 4.88 \\
\hline 7005 & [Ar v] & 0.663 & 0.348 \\
\hline 7136 & [Ar III] & 21.4 & 11.2 \\
\hline 7236 & [Ar IV] & 0.146 & 0.0767 \\
\hline 7319 & [O II] & 6.73 & 3.53 \\
\hline 7330 & [O II] & 5.99 & 3.15 \\
\hline
\end{tabular}

(1) Tsamis et al. (2003). These intensities are integrated over the entire nebula.

size as discussed above. The central star of this nebula is weak and no clear trace of the central star are seen in these spectra.

\subsubsection{IC 4191}

There are no IUE spectra of this nebula. This makes it impossible to determine carbon abundances for this nebula.

\section{Chemical composition of the nebulae}

The method of analysis is the same as used in the papers cited in the introduction. First the electron density and temperature as function of the ionization potential are determined. Then the ionic abundances are determined, using density and temperature appropriate for the ion under consideration. Then the element abundances are found for those elements in which a sufficient number of ionic abundances have been derived.
Table 7. IUE spectrum of NGC 2022.

\begin{tabular}{|c|c|c|c|c|}
\hline \multirow{2}{*}{$\begin{array}{c}\lambda \\
(\AA)\end{array}$} & \multirow[t]{2}{*}{ Ion } & \multicolumn{3}{|c|}{ Intensities } \\
\hline & & (1) & (2) & $(I / \mathrm{H} \beta)$ \\
\hline 1240 & $\mathrm{NV}$ & 4.7 & 4.79 & 61.5 \\
\hline 1400 & O IV] & 11.1 & 8.39 & 108. \\
\hline 1485 & N IV] & 6.4 & 4.49 & 57.7 \\
\hline 1549 & C IV & 99.6 & 70.0 & 900. \\
\hline 1575 & {$[\mathrm{Ne} \mathrm{v}]$} & 3.2 & 2.2 & 29. \\
\hline 1602 & [Ne IV] & 3.1 & 2.2 & 28. \\
\hline 1640 & He II & 78.0 & 52.1 & 670. \\
\hline 1663 & O III] & 3.1 & 2.08 & 26.3 \\
\hline 1750 & N III] & 3.8 & 2.46 & 31.6 \\
\hline 1907.0 & $\mathrm{C}$ III $]^{*}$ & 34.7 & & \\
\hline 1909.1 & $\mathrm{C}$ III] $]^{*}$ & 23.4 & & \\
\hline 1909 & C III] & 69.4 & 47.6 & 612. \\
\hline 2308 & $?$ & 4.7 & & \\
\hline 2423 & [Ne IV] & 39.9 & 25.8 & 323. \\
\hline 2512 & He II & 3.4 & 1.92 & 24.7 \\
\hline 2733 & He II & 5.5 & 2.43 & 31.3 \\
\hline 3046 & O III & 3.5 & & \\
\hline 3130 & O III & 19.9 & & \\
\hline 3200 & He II & 10.5 & 3.7 & 47.6 \\
\hline 3425 & {$[\mathrm{Ne} \mathrm{v}]$} & $38 .^{* *}$ & 12.35 & 69. \\
\hline
\end{tabular}

(1) Measured intensity from low resolution spectra in units of $10^{-13} \mathrm{erg} \mathrm{cm}^{-2} \mathrm{~s}^{-1}$.

(2) Intensity corrected for extinction in units of $10^{-12} \mathrm{erg} \mathrm{cm}^{-2} \mathrm{~s}^{-1}$. $I / \mathrm{H} \beta$ normalized to $\mathrm{H} \beta=100$.

${ }^{*}$ From high resolution spectrum; ${ }^{* *}$ from Rowlands et al. (1993).

\subsection{Electron density}

The ions used to determine $N_{\mathrm{e}}$ are listed in the first column of Table 9 (for NGC 2022), Table 10 (for NGC 6818), and Table 11 (for IC 4191). The ionization potential required to reach that ionization stage, and the wavelengths of the lines used, are given in Cols. 2 and 3 of the tables. Note that the wavelength units are $\AA$ when 4 ciphers are given and microns when 3 ciphers are shown. The observed ratio of the lines is given in the fourth column; the corresponding $N_{\mathrm{e}}$ is given in the fifth column. The temperature used is discussed in the following section, but is unimportant since these line ratios are essentially determined by the density.

There is no indication that the electron density varies with ionization potential in a systematic way in either of the nebulae. As already pointed out by Liu et al. (2001) the [O III] lines always give a lower density than the other lines. Ignoring these lines, the electron density appears to be about $1000 \mathrm{~cm}^{-3}$ for NGC 2022, about $2000 \mathrm{~cm}^{-3}$ for NGC 6818 and $10000 \mathrm{~cm}^{-3}$ IC 4191 . The error is about $20 \%$. It is interesting to compare this value of the density with the rms density found from the $\mathrm{H} \beta$ line. This depends on the distance of the nebula which isn't accurately known, and on the angular size of the nebula. For this calculation we shall use a distance of $1.5 \mathrm{kpc}$ for NGC 2022, $2 \mathrm{kpc}$ for NGC 6818 and $2.1 \mathrm{kpc}$ for IC 4191. 
Table 8. IUE spectrum of NGC 6818.

\begin{tabular}{clccc}
\hline \hline$\lambda$ & Ion & \multicolumn{2}{c}{ Intensities } & I/H $\beta$ \\
\cline { 3 - 4 }$(\AA)$ & & $(1)$ & $(2)$ & $(3)$ \\
\hline 1240 & N V & 1.31 & 7.9 & 19.5 \\
1401 & O IV] & 4.53 & 21.5 & 42.2 \\
1485 & N IV] & 4.93 & 22.2 & 41.4 \\
1550 & C IV & 15.7 & 70.8 & 130. \\
1640 & He II & 53.8 & 242. & 422. \\
1663 & O III] & 5.12 & 22.8 & 39.6 \\
1750 & N III] & 3.91 & 16.4 & 28.3 \\
1883 & Si III] & $1.9:$ & $7.8:$ & 15.8 \\
1909 & C III] & 73.2 & 319. & 600. \\
2320 & O III] & 0.47 & 2.4 & 5.5 \\
2325 & C II] & 3.24 & 16.5 & 33.1 \\
2424 & [Ne IV] & 17.0 & 71.3 & 123. \\
2511 & He II & 1.71 & 6.5 & 10.0 \\
2733 & He II & 3.21 & 10.0 & 13.0 \\
2785 & [Mg V] & 1.5 & 4.9 & 6.2 \\
2836 & O III & 2.77 & 8.3 & 11.0 \\
3024 & O III & 1.4 & 4.2 & 4.8 \\
3047 & O III & 0.7 & 2.1 & 2.4 \\
3133 & O III & 25.4 & 67.9 & 76.0 \\
3203 & He II & 7.0 & 24.5 & 26.5 \\
\hline & & & & \\
\hline
\end{tabular}

(1), (2) I(lines) in intensity units $10^{-12} \mathrm{erg} \mathrm{cm}^{-2} \mathrm{~s}^{-1}$.

(3) $I($ line $) / I(\mathrm{H} \beta)$ corrected for extinction, normalized to $\mathrm{H} \beta=100$.

These distances are taken from (an average of) the individual distances listed by Acker et al. (1992). We do not use statistical values because we do not wish to assume that all nebulae have the same evolution. A sphere of radius of 9.5" will represent NGC 2022, a radius of $10^{\prime \prime}$ will represent NGC 6818, and 2.5" will represent IC 4191. These values are slightly lower than the sizes given in the introduction and are meant to represent values from which most of the flux originates. These are very uncertain values. The $\mathrm{H} \beta$ flux has been given above and the electron temperature will be discussed below. We obtain the uncertain rms values of $1200 \mathrm{~cm}^{-3}$ for NGC 2022, and $1900 \mathrm{~cm}^{-3}$ for NGC 6818 and 11000 for IC 4191. In spite of the uncertainties involved in these calculations (the uncertain distance and the assumption of a spherical homogeneous nebula), there is a strong similarity of these values to the forbidden line densities. This probably indicates that inhomogeneities do not play a dominant role in determining the density. We will use the forbidden line densities in further discussion of the abundances.

\subsection{Electron temperature}

A number of ions have lines originating from energy levels far enough apart that their ratio is sensitive to the electron temperature. These are listed in Tables 12-14, which are arranged similarly to the previous tables. The electron temperature for NGC 2022 and NGC 6818 increase somewhat as a function of ionization. This is not the case for IC 4191, where the
Table 9. Electron density indicators in NGC 2022.

\begin{tabular}{lcccc}
\hline \hline Ion & $\begin{array}{c}\text { Ioniz. } \\
\text { Pot. }(\mathrm{eV})\end{array}$ & $\begin{array}{c}\text { Lines } \\
\text { used }\end{array}$ & $\begin{array}{c}\text { Observed } \\
\text { ratio }\end{array}$ & $\begin{array}{c}N_{\mathrm{e}} \\
\left(\mathrm{cm}^{-3}\right)\end{array}$ \\
\hline$[\mathrm{S} \mathrm{II}]$ & 10.4 & $6731 / 6716$ & 1.12 & 1000 \\
{$[\mathrm{O}$ II $]$} & 13.6 & $3626 / 3729$ & 1.17 & 1000 \\
{$[\mathrm{Cl}$ III $]$} & 23.8 & $5538 / 5518$ & 0.835 & 1100 \\
{$[\mathrm{C}$ III $]$} & 24.4 & $1907 / 1909$ & 1.48 & $\leq 1000$ \\
{$[\mathrm{O}$ III $]$} & 35.1 & $51.9 / 88.4$ & 2.36 & 900 \\
{$[\mathrm{Ar}$ IV $]$} & 40.7 & $4740 / 4711$ & 0.857 & 1300 \\
\hline
\end{tabular}

Table 10. Electron density indicators in NGC 6818.

\begin{tabular}{lcccc}
\hline \hline Ion & $\begin{array}{c}\text { Ioniz. } \\
\text { Pot. }(\mathrm{eV})\end{array}$ & $\begin{array}{c}\text { Lines } \\
\text { used }\end{array}$ & $\begin{array}{c}\text { Observed } \\
\text { ratio }\end{array}$ & $\begin{array}{c}N_{\mathrm{e}} \\
\left(\mathrm{cm}^{-3}\right)\end{array}$ \\
\hline$[\mathrm{S} \mathrm{II}]$ & 10.4 & $6731 / 6716$ & 1.35 & 1870 \\
{$[\mathrm{O}$ II $]$} & 13.6 & $3626 / 3729$ & 1.466 & 2000 \\
{$[\mathrm{~S} \mathrm{III}]$} & 23.3 & $33.5 / 18.7$ & 0.52 & 2300 \\
{$[\mathrm{Cl}$ III $]$} & 23.8 & $5538 / 5518$ & 0.972 & 1900 \\
{$[\mathrm{C} \mathrm{III}]$} & 24.4 & $1907 / 1909$ & 1.42 & 2500 \\
{$[\mathrm{O}$ III $]$} & 35.1 & $51.9 / 88.4$ & 2.36 & 950 \\
{$[$ Ar IV $]$} & 40.7 & $4711 / 4740$ & 1.07 & 2100 \\
{$[\mathrm{Ne}$ IV $]$} & 63.5 & $2425 / 2422$ & 0.987 & 4500 \\
\hline
\end{tabular}

Table 11. Electron density indicators in IC 4191.

\begin{tabular}{lcccc}
\hline \hline Ion & $\begin{array}{c}\text { Ioniz. } \\
\text { Pot. }(\mathrm{eV})\end{array}$ & $\begin{array}{c}\text { Lines } \\
\text { used }\end{array}$ & $\begin{array}{c}\text { Observed } \\
\text { ratio }\end{array}$ & $\begin{array}{c}N_{\mathrm{e}} \\
\left(\mathrm{cm}^{-3}\right)\end{array}$ \\
\hline$[\mathrm{S} \mathrm{II}]$ & 10.4 & $6731 / 6716$ & 1.87 & 7400 \\
{$[\mathrm{~S} \mathrm{III}]$} & 23.3 & $33.5 / 18.7$ & 0.199 & 9000 \\
{$[\mathrm{Cl}$ III $]$} & 23.8 & $5538 / 5518$ & 1.85 & 12000 \\
{$[\mathrm{Ar}$ III $]$} & 27.6 & $21.8 / 8.99$ & 0.064 & 7000 \\
{$[\mathrm{O} \mathrm{III}]$} & 35.1 & $51.9 / 88.4$ & 3.61 & 2000 \\
{$[\mathrm{Ar}$ IV $]$} & 40.7 & $4740 / 4711$ & 1.70 & 12000 \\
\hline
\end{tabular}

temperature clearly remains approximately constant as a function of ionization potential. The results of the [Ar IV] line ratio has been ignored because of the uncertainty in the line intensities. In all the nebulae the results of the [Ne III] line ratio gives a lower temperature than the other lines. This has already been noticed in many nebulae (see references in Sect. 1).

\subsection{Ionic and element abundances}

The ionic abundances have been determined using the following equation:

$\frac{N_{\text {ion }}}{N_{\mathrm{p}}}=\frac{I_{\text {ion }}}{I_{\mathrm{H}_{\beta}}} N_{\mathrm{e}} \frac{\lambda_{\mathrm{ul}}}{\lambda_{\mathrm{H}_{\beta}}} \frac{\alpha_{\mathrm{H}_{\beta}}}{A_{\mathrm{ul}}}\left(\frac{N_{\mathrm{u}}}{N_{\text {ion }}}\right)^{-1}$

where $I_{\text {ion }} / I_{\mathrm{H}_{\beta}}$ is the measured intensity of the ionic line compared to $\mathrm{H} \beta, N_{\mathrm{p}}$ is the density of ionized hydrogen, $\lambda_{\mathrm{ul}}$ is the wavelength of this line, $\lambda_{\mathrm{H}_{\beta}}$ is the wavelength of $\mathrm{H} \beta, \alpha_{\mathrm{H}_{\beta}}$ is the effective recombination coefficient for $\mathrm{H} \beta, A_{\mathrm{ul}}$ is the Einstein spontaneous transition rate for the line, and $N_{\mathrm{u}} / N_{\text {ion }}$ is the ratio 
Table 12. Electron temperature indicators in NGC 2022.

\begin{tabular}{lcccc}
\hline \hline Ion & $\begin{array}{c}\text { Ioniz. } \\
\text { Pot. }(\mathrm{eV})\end{array}$ & $\begin{array}{c}\text { Lines } \\
\text { used }\end{array}$ & $\begin{array}{c}\text { Observed } \\
\text { ratio }\end{array}$ & $\begin{array}{c}T_{\mathrm{e}} \\
(\mathrm{K})\end{array}$ \\
\hline$[\mathrm{S} \mathrm{III}]$ & 23.3 & $6312 / 18.7$ & 0.107 & 13900 \\
{$[\mathrm{Ar}$ III $]$} & 27.6 & $7136 / 8.99$ & 0.82 & 8900 \\
{$[\mathrm{~N} \mathrm{III}]$} & 29.6 & $1750 / 57.4$ & 0.94 & 13400 \\
{$[\mathrm{O}$ III $]$} & 35.1 & $4363 / 5007$ & 0.018 & 14300 \\
{$[\mathrm{O} \mathrm{III}]$} & 35.1 & $1663 / 5007$ & 0.0376 & 14100 \\
{$[\mathrm{Ne} \mathrm{III}]$} & 41.0 & $3869 / 15.5$ & 0.91 & 11500 \\
{$[\mathrm{O}$ IV $]$} & 54.9 & $1400 / 25.8$ & 0.069 & 16000 \\
{$[\mathrm{Ar}$ V $]$} & 59.8 & $7005 / 7.9$ & 0.36 & 14800 \\
{$[\mathrm{Ne}$ IV $]$} & 63.5 & $4725 / 2423$ & 0.0058 & 15000 \\
{$[\mathrm{Ne}$ $]$} & 97.1 & $3425 / 14.3$ & 0.051 & 19000 \\
\hline
\end{tabular}

Table 13. Electron temperature indicators in NGC 6818.

\begin{tabular}{lcccc}
\hline \hline Ion & $\begin{array}{c}\text { Ioniz. } \\
\text { Pot. }(\mathrm{eV})\end{array}$ & $\begin{array}{c}\text { Lines } \\
\text { used }\end{array}$ & $\begin{array}{c}\text { Observed } \\
\text { ratio }\end{array}$ & $\begin{array}{c}T_{\mathrm{e}} \\
(\mathrm{K})\end{array}$ \\
\hline$[\mathrm{N} \mathrm{II}]$ & 14.5 & $5755 / 6584$ & 0.0197 & 10600 \\
{$[\mathrm{~S} \mathrm{III}]$} & 23.3 & $6312 / 18.7$ & 0.0933 & 12800 \\
{$[\mathrm{~S} \mathrm{III}]$} & 23.3 & $6312 / 9531$ & 0.042 & 13000 \\
{$[\mathrm{Ar} \mathrm{III}]$} & 27.6 & $7136 / 8.99$ & $1.5:$ & 13000 \\
{$[\mathrm{Ar}$ III $]$} & 27.6 & $5192 / 8.99$ & 0.201 & 12500 \\
{$[\mathrm{~N} \mathrm{III}]$} & 29.6 & $57.4 / 1750$ & 1.08 & 12700 \\
{$[\mathrm{O} \mathrm{III}]$} & 35.1 & $4363 / 5007$ & 0.0151 & 13700 \\
{$[\mathrm{O}$ III $]$} & 35.1 & $1663 / 5007$ & 0.0291 & 13500 \\
{$[\mathrm{Ne} \mathrm{III}]$} & 41.0 & $3868 / 15.5$ & 1.08 & 11700 \\
{$[\mathrm{O}$ IV $]$} & 54.7 & $1400 / 25.9$ & 0.0567 & 15300 \\
{$[\mathrm{Ne} \mathrm{V}]$} & 97.1 & $3425 / 24.3$ & 0.382 & 16000 \\
{$[\mathrm{Ne}$ $]$} & 97.1 & $3425 / 14.3$ & 0.501 & 18700 \\
{$[\mathrm{Mg} \mathrm{v}]$} & 109.3 & $2785 / 13.5$ & 7.0 & 16900 \\
\hline
\end{tabular}

Table 14. Electron temperature indicators in IC 4191.

\begin{tabular}{lcccc}
\hline \hline Ion & $\begin{array}{c}\text { Ioniz. } \\
\text { Pot. }(\mathrm{eV})\end{array}$ & $\begin{array}{c}\text { Lines } \\
\text { used }\end{array}$ & $\begin{array}{c}\text { Observed } \\
\text { ratio }\end{array}$ & $\begin{array}{c}T_{\mathrm{e}} \\
(\mathrm{K})\end{array}$ \\
\hline$[\mathrm{N} \mathrm{II}]$ & 14.5 & $5755 / 6584$ & 0.028 & 11000 \\
{$[\mathrm{~S} \mathrm{III}]$} & 23.3 & $6312 / 18.7$ & 0.0604 & 9500 \\
{$[\mathrm{Ar}$ III $]$} & 27.6 & $5192 / 8.99$ & 0.0059 & 9800 \\
{$[\mathrm{Ar}$ III $]$} & 27.6 & $7136 / 8.99$ & 0.735 & 8000 \\
{$[\mathrm{O} \mathrm{III}]$} & 35.1 & $4363 / 5007$ & 0.0067 & 10000 \\
{$[\mathrm{O} \mathrm{III}]$} & 35.1 & $5007 / 51.9$ & 16.1 & 9600 \\
{$[\mathrm{Ne}$ III $]$} & 41.0 & $3868 / 15.5$ & 0.332 & 8500 \\
{$[\mathrm{Ar}$ IV $]$} & 41.0 & $7263 / 4711$ & 0.447 & 15000 \\
\hline
\end{tabular}

of the population of the level from which the line originates to the total population of the ion. This ratio has been determined using a five level atom.
Table 15. Ionic concentrations and chemical abundances in NGC 2022. Wavelength in Angstrom for all values of $\lambda$ above 1000, otherwise in $\mu \mathrm{m}$.

\begin{tabular}{|c|c|c|c|c|c|c|}
\hline Ion & $\lambda$ & $T_{\mathrm{e}}$ & $I / \mathrm{H} \beta$ & $N_{\text {ion }} / N_{\mathrm{p}}$ & ICF & $N_{\text {el. }} / N_{\mathrm{p}}$ \\
\hline $\mathrm{He}^{+}$ & 5875 & 13500 & 1.90 & 0.012 & & \\
\hline $\mathrm{He}^{++}$ & 4686 & 15000 & 108. & 0.094 & 1 & 0.106 \\
\hline $\mathrm{C}^{++}$ & 1909 & 13500 & 612. & $2.31(-4)$ & & \\
\hline $\mathrm{C}^{+3}$ & 1549 & 14500 & 900. & $1.35(-4)$ & 1 & $3.66(-4)$ \\
\hline $\mathrm{N}^{+}$ & 6584 & 13000 & 2.2 & $2.31(-7)$ & & \\
\hline $\mathrm{N}^{++}$ & 1750 & 14000 & 31.6 & $3.65(-5)$ & & \\
\hline $\mathrm{N}^{++}$ & 57 & 14000 & 33.6 & $4.69(-5)$ & & \\
\hline $\mathrm{N}^{+3}$ & 1485 & 15500 & 57.7 & $3.65(-5)$ & & \\
\hline $\mathrm{N}^{+4}$ & 1240 & 16500 & 61.5 & $1.83(-5)$ & 1 & $9.9(-5)$ \\
\hline $\mathrm{O}^{+}$ & 3727 & 12000 & 14. & $2.58(-6)$ & & \\
\hline $\mathrm{O}^{++}$ & 5007 & 14500 & 720. & $9.62(-5)$ & & \\
\hline $\mathrm{O}^{++}$ & 1663 & 14500 & 26.3 & $7.96(-5)$ & & \\
\hline $\mathrm{O}^{+3}$ & 1400 & 15500 & 108. & $2.86(-4)$ & & \\
\hline $\mathrm{O}^{+3}$ & 25.8 & 15000 & 1565. & $3.08(-4)$ & 1.2 & $4.74(-4)$ \\
\hline $\mathrm{Ne}^{+}$ & 12.8 & 13000 & 10.3 & $1.19(-5)$ & & \\
\hline $\mathrm{Ne}^{++}$ & 15.5 & 14500 & 56.8 & $3.29(-5)$ & & \\
\hline $\mathrm{Ne}^{++}$ & 3869 & 14500 & 53.0 & $1.64(-5)$ & & \\
\hline $\mathrm{Ne}^{+3}$ & 2423 & 15500 & 332. & $7.61(-5)$ & & \\
\hline $\mathrm{Ne}^{+4}$ & 14.3 & 17500 & 134. & $1.13(-5)$ & & \\
\hline $\mathrm{Ne}^{+4}$ & 24.3 & 17500 & 172. & $1.50(-5)$ & 1 & $1.34(-4)$ \\
\hline $\mathrm{Mg}^{+3}$ & 4.49 & 16500 & 29.3 & $1.18(-5)$ & & \\
\hline $\mathrm{Mg}^{+4}$ & 5.56 & 18000 & 18.1 & $4.17(-6)$ & 3: & $4.8(-5)$ \\
\hline $\mathrm{S}^{+}$ & 6731 & 12500 & 0.73 & $2.13(-8)$ & & \\
\hline $\mathrm{S}^{++}$ & 18.7 & 13500 & 16.9 & $1.32(-6)$ & & \\
\hline $\mathrm{S}^{++}$ & 6312 & 13500 & 1.8 & $1.37(-6)$ & & \\
\hline $\mathrm{S}^{+3}$ & 10.5 & 14500 & 160. & $3.61(-6)$ & 1.25 & $6.3(-6)$ \\
\hline $\mathrm{Ar}^{++}$ & 8.99 & 13800 & 11.6 & $9.9(-7)$ & & \\
\hline $\mathrm{Ar}^{++}$ & 7136 & 13800 & 9.5 & $4.68(-7)$ & & \\
\hline $\mathrm{Ar}^{+3}$ & 4740 & 14500 & 12.1 & $1.38(-6)$ & & \\
\hline $\mathrm{Ar}^{+4}$ & 7005 & 15600 & 3.5 & $3.28(-7)$ & & \\
\hline $\mathrm{Ar}^{+4}$ & 7.9 & 15600 & 6.35 & $2.47(-7)$ & 1 & $2.7(-6)$ \\
\hline $\mathrm{Cl}^{++}$ & 5538 & 13400 & 0.36 & $2.47(-8)$ & & \\
\hline $\mathrm{Cl}^{+3}$ & 8045 & 14500 & 1.3 & $6.26(-8)$ & 1.5: & 1.3(-7): \\
\hline $\mathrm{K}^{+3}$ & 6102 & 15000 & 0.64 & $2.42(-8)$ & $3:$ & 7.2(-8): \\
\hline $\mathrm{C}^{++}$ & 4267 & 13000 & 0.82 & $9.4(-4)$ & & \\
\hline
\end{tabular}

Intensities given with respect to $\mathrm{H} \beta=100$.

(:) Indicates uncertain value.

\subsubsection{NGC 2022}

The results are given in Table 15, where the first column lists the ion concerned, and the second column the line used for the abundance determination. The third column gives the intensity of the line used relative to $\mathrm{H} \beta=100$. The fourth column gives the ionic abundances, and the fifth column gives the Ionization Correction Factor (ICF). This has been determined empirically. Notice that the ICF is unity (or almost unity) and the element abundances, given in the last column, are probably well 
determined. The carbon recombination line abundances are given at the end of the table.

The abundances in NGC 2022 are in general very similar to solar abundances. This will be discussed in detail presently. The abundances of oxygen, nitrogen, neon and argon are close to those of the sun. Sulfur is lower than solar, but this is true in most nebulae and it is likely that the solar abundance is anomalous. Carbon is slightly higher than the solar abundance but only by $50 \%$.

\subsubsection{NGC 6818}

The results are given in Table 16 which has the same format as the previous table.

The abundances in NGC 6818 resemble those of NGC 2022 in that oxygen, helium, neon and argon are essentially the same and nitrogen is quite similar. Carbon is somewhat higher, however, so that C/O is somewhat greater than unity in NGC 6818 while it is less than unity in NGC 2022.

\subsubsection{IC 4191}

The results are given in Table 17 which has the same format as the previous two tables. No carbon abundance can be determined since no ultraviolet spectra have been observed. Helium is clearly higher than solar. Oxygen and nitrogen are also about $60 \%$ higher than solar, but the N/O ratio is close to solar. All other elements are about a factor of two greater than in the sun. The electron temperature appears to be roughly constant in this nebula and the abundances have been determined with a value of $T_{\mathrm{e}}=10000 \mathrm{~K}$ and $N_{\mathrm{e}}=10^{-4} \mathrm{~cm}^{-3}$.

\section{Comparison with other abundance determinations}

Tables 18-20 show a comparison of our abundances with the most important determinations in the past 20 years. There is marginal agreement, usually to within a factor of two or three. It is rather surprising that such differences exist, even for oxygen. A comparison is also made with the solar abundance (Anders \& Grevesse 1989; Grevesse \& Sauval 1998; and Allende Prieto et al. 2001, 2002). Note that for sulfur and chlorine more weight has been given to the abundance determination in meteorites since this determination is more accurate than for the sun itself.

The helium abundance has been derived using the theoretical work of Benjamin et al. (1999). For recombination of singly ionized helium, most weight is given to the $\lambda 5875 \AA$ line, because the theoretical determination of this line is the most reliable.

\subsection{Errors}

The errors have been discussed in a general way in the papers cited earlier concerning the ISO spectra and especially in the recent paper (Pottasch et al. 2004). These general considerations are applicable here as well. Therefore here we will discuss the reasons for the abundance differences between what
Table 16. Ionic concentrations and chemical abundances in NGC 6818. Wavelength in Angstrom for all values of $\lambda$ above 1000, otherwise in $\mu \mathrm{m}$.

\begin{tabular}{|c|c|c|c|c|c|c|}
\hline Ion & $\lambda$ & $T_{\mathrm{e}}$ & $I / \mathrm{H} \beta$ & $N_{\text {ion }} / N_{\mathrm{p}}$ & ICF & $N_{\mathrm{el} .} / N_{\mathrm{p}}$ \\
\hline $\mathrm{He}^{+}$ & 5875 & 12000 & 7.46 & 0.045 & & \\
\hline $\mathrm{He}^{++}$ & 4686 & 15500 & 62. & 0.054 & 1 & 0.099 \\
\hline $\mathrm{C}^{+}$ & 2325 & 10500 & 33.1 & $4.35(-5)$ & & \\
\hline $\mathrm{C}^{++}$ & 1909 & 12100 & 600. & $4.1(-4)$ & & \\
\hline $\mathrm{C}^{+3}$ & 1549 & 15000 & 130. & $8.1(-5)$ & 1 & $5.4(-4)$ \\
\hline $\mathrm{N}^{+}$ & 6584 & 10500 & 62.5 & $9.8(-6)$ & & \\
\hline $\mathrm{N}^{++}$ & 1750 & 12750 & 28.3 & $6.2(-5)$ & & \\
\hline $\mathrm{N}^{++}$ & 57 & 12750 & 34.7 & $8.07(-5)$ & & \\
\hline $\mathrm{N}^{+3}$ & 1485 & 15000 & 41.4 & $3.5(-5)$ & & \\
\hline $\mathrm{N}^{+4}$ & 1240 & 16500 & 19.5 & $6.3(-6)$ & 1 & $1.26(-4)$ \\
\hline $\mathrm{O}^{+}$ & 3726 & 11000 & 35. & $1.85(-5)$ & & \\
\hline $\mathrm{O}^{++}$ & 5007 & 13500 & 1540. & $2.54(-4)$ & & \\
\hline $\mathrm{O}^{++}$ & 51.8 & 13500 & 167. & $1.82(-4)$ & & \\
\hline $\mathrm{O}^{++}$ & 1663 & 13500 & 39.6 & $2.10(-4)$ & & \\
\hline $\mathrm{O}^{+3}$ & 25.8 & 15700 & 843. & $2.17(-4)$ & & \\
\hline $\mathrm{O}^{+3}$ & 1400 & 15700 & 42.2 & $1.43(-4)$ & 1 & $4.8(-4)$ \\
\hline $\mathrm{Ne}^{+}$ & 12.8 & 11500 & 2.68 & $3.39(-6)$ & & \\
\hline $\mathrm{Ne}^{++}$ & 15.5 & 14300 & 131. & $8.30(-5)$ & & \\
\hline $\mathrm{Ne}^{++}$ & 3869 & 14300 & 125. & $4.27(-5)$ & & \\
\hline $\mathrm{Ne}^{+3}$ & 2425 & 16200 & 123. & $2.67(-5)$ & & \\
\hline $\mathrm{Ne}^{+4}$ & 24.3 & 16800 & 131. & $1.41(-5)$ & 1 & $1.27(-4)$ \\
\hline $\mathrm{Mg}^{+3}$ & 4.49 & 16700 & 21.4 & $3.88(-5)$ & & \\
\hline $\mathrm{Mg}^{+4}$ & 13.5 & 17000 & 0.996 & $3.03(-6)$ & 3: & $1.26(-4)$ \\
\hline$S^{+}$ & 6731 & 10000 & 7.1 & $3.32(-7)$ & & \\
\hline $\mathrm{S}^{++}$ & 18.7 & 12000 & 37.5 & $3.26(-6)$ & & \\
\hline $\mathrm{S}^{++}$ & 6312 & 12000 & 3.3 & $5.06(-6)$ & & \\
\hline$S^{+3}$ & 10.5 & 13500 & 133. & $3.20(-6)$ & 1.25 & $9.4(-6)$ \\
\hline $\mathrm{Ar}^{++}$ & 8.99 & 12500 & 15.4 & $1.4(-6)$ & & \\
\hline $\mathrm{Ar}^{+3}$ & 4740 & 14300 & 7.4 & $9.07(-7)$ & & \\
\hline $\mathrm{Ar}^{+4}$ & 7.90 & 16000 & 3.09 & $1.32(-7)$ & & \\
\hline $\mathrm{Ar}^{+4}$ & 7005 & 16000 & 1.36 & $1.34(-7)$ & 1 & $2.7(-6)$ \\
\hline $\mathrm{Cl}^{++}$ & 5538 & 12000 & 0.87 & $7.54(-8)$ & & \\
\hline $\mathrm{Cl}^{+3}$ & 8045 & 14200 & 1.8 & $9.58(-8)$ & 1.5: & $2.6(-7)$ \\
\hline $\mathrm{C}^{++}$ & 4267 & 12100 & 0.45 & $5.1(-4)$ & & \\
\hline
\end{tabular}

Intensities given with respect to $\mathrm{H} \beta=100$.

(:) Indicates uncertain values.

we have obtained and what has been found by Tsamis et al. (2003). This is interesting because we have used essentially the same visual measurements as this group, and the comparison provides some insight into the reasons for the differences. The ultraviolet measurements are somewhat different however. The measurements of NGC 2022 have been rereduced, but in general do not differ substantially from those used by Tsamis et al. The ultraviolet measurements of NGC 6818 are the same as used by Tsamis et al. except that the normalization is somewhat different. We use here a He II $\lambda 1640 / \lambda 4686$ ratio of 6.81 which is the theoretical value given by Hummer \& Storey (1987). 
Table 17. Ionic concentrations and chemical abundances in IC 4191. Wavelength in Angstrom for all values of $\lambda$ above 1000, otherwise in $\mu \mathrm{m}$.

\begin{tabular}{lccccc}
\hline \hline Ion & $\lambda$ & Intens./H $\beta$ & $N_{\text {ion }} / N_{\mathrm{p}}$ & ICF & $N_{\text {el. } /} / N_{\mathrm{p}}$ \\
\hline $\mathrm{He}^{+}$ & 5875 & 17.2. & 0.112 & & \\
$\mathrm{He}^{++}$ & 4686 & 13.5 & 0.011 & 1 & 0.123 \\
$\mathrm{~N}^{+}$ & 6584 & 80. & $1.72(-5)$ & & \\
$\mathrm{N}^{++}$ & 57 & 9.7 & $1.15(-4)$ & 1.1 & $1.49(-4)$ \\
$\mathrm{O}^{+}$ & 3726 & 58. & $7.64(-5)$ & & \\
$\mathrm{O}^{++}$ & 5007 & 1500. & $5.49(-4)$ & & \\
$\mathrm{O}^{++}$ & 51.8 & 93.2 & $6.47(-4)$ & 1 & $6.6(-4)$ \\
$\mathrm{Ne}^{++}$ & 15.5 & 416. & $2.9(-4)$ & & \\
$\mathrm{Ne}^{++}$ & 3869 & 136. & $1.37(-4)$ & & \\
$\mathrm{Ne}^{+3}$ & 4724 & 0.18 & $2.0(-4)$ & & \\
$\mathrm{Ne}^{+3}$ & 4725 & 0.125 & $1.6(-4)$ & 1 & $4.7(-4)$ \\
$\mathrm{Mg}^{+3}$ & 4.49 & 8.1 & $4.1(-6)$ & & \\
$\mathrm{S}^{+}$ & 6731 & 9.0 & $8.49(-7)$ & & \\
$\mathrm{S}^{++}$ & 18.7 & 42.9 & $7.31(-6)$ & & \\
$\mathrm{S}^{++}$ & 6312 & 2.61 & $5.58(-6)$ & & \\
$\mathrm{S}^{+3}$ & 10.5 & 200. & $8.11(-6)$ & 1 & $1.6(-5)$ \\
$\mathrm{Ar}^{++}$ & 8.99 & 29.1 & $3.05(-6)$ & & \\
$\mathrm{Ar}^{++}$ & 7136 & 20.0 & $1.83(-6)$ & & \\
$\mathrm{Ar}^{+3}$ & 4740 & 5.3 & $1.31(-6)$ & & \\
$\mathrm{Ar}^{+4}$ & 7005 & 0.663 & $1.37(-7)$ & 1 & $4.45(-6)$ \\
$\mathrm{Cl}^{++}$ & 5538 & 0.856 & $1.11(-7)$ & $2:$ & $2.2(-7)$ \\
$\mathrm{K}^{+3}$ & 6101 & 0.28 & $2.38(-8)$ & & \\
$\mathrm{C}^{++}$ & 4267 & 0.546 & $6.0(-4)$ & & \\
\hline & & & & & \\
\hline
\end{tabular}

Intensities given with respect to $\mathrm{H} \beta=100$.

Table 18. Comparison of abundances in NGC 2022.

\begin{tabular}{lrrrr}
\hline \hline Elem. & Present & Tsamis & AC 83 & Solar \\
\hline $\mathrm{He}$ & 0.106 & 0.11 & 0.108 & 0.098 \\
$\mathrm{C}(-4)$ & 3.66 & 2.15 & 3.5 & 2.5 \\
$\mathrm{~N}(-5)$ & 9.9 & 2.9 & 7.6 & 8.4 \\
$\mathrm{O}(-4)$ & 4.74 & 4.6 & 1.95 & 4.9 \\
$\mathrm{Ne}(-4)$ & 1.34 & 0.70 & 1.1 & 1.2 \\
$\mathrm{~S}(-6)$ & 6.3 & 3.7 & $32 .:$ & 16. \\
$\mathrm{Ar}(-6)$ & 2.7 & 1.3 & 3.3 & 2.5 \\
$\mathrm{Cl}(-7)$ & 1.3 & 0.62 & 3.0 & 1.9 \\
\hline
\end{tabular}

References: Tsamis et al. (2003); AC 83: Aller \& Czyzak (1983). Solar: Anders \& Grevesse (1989); Allende Prieto et al. (2001, 2002); Grevesse \& Sauval (1998).

Tsamis et al. use a value of 5.20. The largest differences arise from the inclusion of the ISO measurements. We will discuss each element separately.

For oxygen we obtain the same abundance as Tsamis et al. This is not so surprising since much of the oxygen is in $\mathrm{O}^{++}$for which the same observations were used and a similar electron temperature derived. The $\mathrm{O}^{3+}$ is also important for NGC 2022 and NGC 6818; we obtain the same abundances within $10 \%$.
Table 19. Comparison of abundances in NGC 6818.

\begin{tabular}{lrrrrr}
\hline \hline Elem. & Present & Tsamis & Hyung & AC 83 & Solar \\
\hline $\mathrm{He}$ & 0.099 & 0.099 & 0.105 & 0.112 & 0.098 \\
$\mathrm{C}(-4)$ & 5.4 & 2.6 & 8.0 & 4.7 & 2.5 \\
$\mathrm{~N}(-4)$ & 1.26 & 0.55 & 4.0 & 2.1 & 0.84 \\
$\mathrm{O}(-4)$ & 4.8 & 5.08 & 7.0 & 5.3 & 4.9 \\
$\mathrm{Ne}(-4)$ & 1.27 & 1.18 & 1.0 & 1.1 & 1.2 \\
$\mathrm{Mg}(-5)$ & 12.6 & & 3.0 & & 3.8 \\
$\mathrm{~S}(-5)$ & 0.94 & 0.36 & 0.70 & 1.1 & 1.6 \\
$\mathrm{Ar}(-6)$ & 2.7 & 0.87 & 4.0 & 3.7 & 2.5 \\
$\mathrm{Cl}(-7)$ & 2.6 & 1.24 & 3.0 & 1.4 & 1.9 \\
\hline
\end{tabular}

References: Tsamis: Tsamis et al. (2003); Hyung: Hyung et al. (1999); AC 83: Aller \& Czyzak (1983); Solar: Anders \& Grevesse (1989); Allende Prieto et al. (2001, 2002); Grevesse \& Sauval (1998).

Table 20. Comparison of abundances in IC 4191.

\begin{tabular}{lrrrr}
\hline \hline Elem. & Present & Tsamis & GMM & Solar \\
\hline $\mathrm{He}$ & 0.123 & 0.121 & 0.11 & 0.098 \\
$\mathrm{~N}(-4)$ & 1.49 & 0.39 & 2.2 & 0.84 \\
$\mathrm{O}(-4)$ & 7.7 & 6.05 & 6.05 & 4.9 \\
$\mathrm{Ne}(-4)$ & 4.7 & 1.62 & 1.45 & 1.2 \\
$\mathrm{~S}(-5)$ & 1.6 & 1.27 & 0.60 & 1.6 \\
$\mathrm{Ar}(-6)$ & 4.45 & 2.5 & & 2.5 \\
$\mathrm{Cl}(-7)$ & 2.2 & 2.35 & & 1.9 \\
\hline
\end{tabular}

References: Tsamis: Tsamis et al. (2003); GMM: Gutierrez-Moreno \& Moreno (1988); Solar: Anders \& Grevesse (1989); Allende Prieto et al. (2001, 2002); Grevesse \& Sauval (1998).

For carbon we find higher abundances than Tsamis et al. for both NGC 2022 and NGC 6818. This is because we find somewhat larger measurements of the ultraviolet carbon lines, and because our electron temperatures in which these ions are formed are about $1000 \mathrm{~K}$ lower.

We get consistently higher abundances for nitrogen by about a factor of three. This mirrors the $\mathrm{N}^{++}$abundance, which is the most abundant ion. Here the difference is that we have made use of the $57 \mu \mathrm{m}$ line, emitted by the lowest excited level of this ion. The abundance from this line is not dependent on the electron temperature, but it is dependent on the electron density, for which we have used the average value discussed above. But we have found higher values of the abundance for the other nitrogen ions as well. For neon we find a higher $\mathrm{Ne}^{++}$abundance from the infrared $15.5 \mu \mathrm{m}$ than from the $\lambda 3869 \AA$ line. This has been found in other nebulae as well. Since we give more weight to the $15.5 \mu \mathrm{m}$ line, which was not used by Tsamis et al., we find a higher neon abundance for two of the three nebulae (NGC 2022 and IC 4191).

The infrared ISO lines also play an important role in the sulfur abundance. We give more weight to the rather strong $18.7 \mu \mathrm{m}$ line of $\mathrm{S}^{++}$than to the weak $\lambda 6312 \AA$ line. And we determine the rather important $\mathrm{S}^{3+}$ abundance using the $10.5 \mu \mathrm{m}$ line. Tsamis et al. have no measurement of this line and only estimate the abundance of this ion. As a result we find a higher 
sulfur abundance. The argon abundance is also found to be higher, mainly due to the use of the $8.99 \mu \mathrm{m}$ line of $\mathrm{Ar}^{++}$. But $\mathrm{Ar}^{3+}$ is also found to be higher due to the somewhat lower electron temperatures we found to be associated with this ion.

\subsection{Comparison with ICF from visual spectrum}

When only the visual spectrum is available for analysis the abundance of only a limited number of ions can be found. To find the total element abundance an ionization correction factor (ICF) is necessary. Much effort has gone into determining what this factor is, and the question arises whether analyses like the present, where many additional ions are observed (thus using UV and far infrared spectra) are able to confirm or better define these values. We have now found abundances for $21 \mathrm{PN}$ and a referee felt that the sample now might be large enough that a discussion could be made.

The discussion given below should only be considered preliminary because the sample is not as large as it seems. Because of the large differences of excitation among the PN considered, we found it necessary to divide them into 4 categories: category $\mathrm{A}$ has no $\mathrm{He}^{++}$; category $\mathrm{B}$ has low $\mathrm{He}^{++}(\leq 10 \%)$; category $\mathrm{C}$ has $\mathrm{He}^{++}$between $10 \%$ and $50 \%$; and category $\mathrm{D}$ has more $\mathrm{He}^{++}$than $\mathrm{He}^{+}$. We compute the abundances from the visual spectrum using the ICF given by Kingsburgh \& Barlow (1994), and limit ourselves to oxygen, nitrogen and neon. In this comparison when a number is given, it is the ratio of the abundance we have found to that computed with only the visual spectrum and the ICF.

Consider the 4 cases in category A. For oxygen the same abundances are found from both methods (within 10\%), for nitrogen we find a slightly higher value (about 1.2 to 1.3 ) but for neon the difference is quite large, between a factor of 1.6 and 6 . This occurs because the ICF method is based on $\mathrm{Ne}^{++}$, which is only a small percentage of the total neon. Category B also has only 4 cases. Here again the same oxygen abundance is found by both methods, and the nitrogen ratio varies from 1 to 1.45 . The neon ratio varies by about the same amount.

In category $\mathrm{C}$ (6 cases) we find somewhat more oxygen that the ICF method, although the ratio varies from about 1.1 to 1.7 , averaging about $30 \%$ higher. The nitrogen ratio varies quite substantially, from about 0.4 to 1.6 , but the average value does not much differ for the two approaches. For neon the ratio varies from 0.8 to 1.9 . In category $\mathrm{D}$ ( 7 cases) larger differences occur. Oxygen is the least affected, the ratio varying from 0.78 to 1.12 . Nitrogen varies between 0.5 and 3.8 and neon between 0.8 and 1.6. We should remark that in the case of neon, $\mathrm{Ne}^{++}$was always determined from the $15.5 \mu \mathrm{m}$ line. Had the $\lambda 3869 \AA$ line been used, as is the case when the visual spectrum is used, the ratio would have sometimes been considerably higher. This is because in some nebulae these two lines give somewhat different results when the electron temperature found from the $\mathrm{O}^{++}$lines is used. This can be seen in Tables $15-17$ of this paper. We have always used the results from the $15.5 \mu \mathrm{m}$ line since this is independent of the electrom temperature.
From this discussion it is not possible to suggest improvements to the ICF. For low excitation objects neon abundances will have to be increased from the ICF value, but a precise number is difficult to ascertain. For other nebulae errors in nitrogen abundance of a factor of two will occur, but it is too early to predict when this will happen.

\subsection{Recombination line abundances of carbon}

The $\mathrm{C}^{++}$population can be obtained from the recombination line $\lambda 4267 \AA$ as well as from the collisionally excited line at $\lambda 1909 \AA$. The advantage of the recombination line is that it is not sensitive to the electron temperature, and is in the visual spectrum as well. It has the disadvantage that it is quite faint and thus difficult to measure accurately. It has been used for at least 30 years, and has been found to give higher $\mathrm{C}^{++}$populations than the collisional line. The reason for this is not clear (e.g. see the discussion of Liu et al. 2000).

In NGC 2022 the $\mathrm{C}^{++}$recombination line $\lambda 4267 \AA$ yields an abundance $\mathrm{C}^{++} / \mathrm{H}$ of $9.4 \times 10^{-4}$, using the effective recombination coefficients given by Davey et al. (2000) for their Case B, which refers to a nebula which is optically thick for the resonance transition. This value is somewhat higher than the value of $2.3 \times 10^{-4}$ obtained from the collisionally excited line. For NGC 6818 the $\lambda 4267 \AA$ yields an abundance of $5.1 \times 10^{-4}$, for Case B. The value obtained from the $\lambda 1909 \AA$ collisionally excited line is $4.15 \times 10^{-4}$ which is essentially the same. For IC 4191 only the $\lambda 4267 \AA$ line has been measured. It yields a value of $\mathrm{C}^{++} / \mathrm{H}=6.0 \times 10^{-4}$ which cannot be compared with the collisional line value since it has not been measured.

To summarize, the $\lambda 4267 \AA$ line give the same value of $\mathrm{C}^{++} / \mathrm{H}$ as the collisional lines at $\lambda 1909 \AA$ for NGC 6818 , and a much higher value for NGC 2022. This indicates to us that the formation of this recombination line is not completely understood.

\section{The central star}

\subsection{Stellar temperature}

Enough information is available to compute both the Zanstra temperature and the Energy Balance temperature of the central star. The Zanstra temperature requires the knowledge of the stellar apparent magnitude, the extinction and the $\mathrm{H} \beta$ flux. The last two quantities have already been given in Sect. 3 . The apparent magnitude $V$ is 15.92 for NGC 2022 (Gathier \& Pottasch 1988), $V=17.02$ for NGC 6818 (Gathier \& Pottasch 1988), and an uncertain value of $V=16.4$ for IC 4191 (Shaw \& Kaler 1989). Assuming that the star radiates as a blackbody the hydrogen Zanstra temperature $\left(T_{\mathrm{z}}(\mathrm{H})\right)$ and the ionized helium Zanstra temperature are given in Cols. 2 and 3 of Table 21. The Energy Balance temperature requires the knowledge of the ratio of the "forbidden" line intensities to $\mathrm{H} \beta$. This value is found by summing the intensities given in Sect. 4 , and is 55 for NGC 2022, 51. for NGC 6818 and greater than 35 for IC 4191 (because the ultraviolet line fluxes were not measured). It probably has a value between 47 and 57 for this nebula, since in many nebulae the ultraviolet lines contribute a value 
Table 21. Central star temperatures.

\begin{tabular}{lcccc}
\hline \hline Nebula & $T_{z}(\mathrm{H})$ & $T_{z}(\mathrm{HeII})$ & $T_{\mathrm{EB}}$ & Temp. \\
\hline NGC 2022 & 59000 & 108000 & 122000 & 122000 \\
NGC 6818 & 140000 & 155000 & 138000 & 145000 \\
IC 4191 & 79000 & 90000 & $\geq 95000$ & 95000 \\
\hline
\end{tabular}

of between 12 and 22. This is a very uncertain number, especially since the carbon abundance (whose lines are an important contributer) is not known. To convert these values to a stellar temperature, the formulation of Preite-Martinez \& Pottasch (1983) is used, assuming blackbody radiation from the central star. The value of Case II (the nebula is optically thin for radiation which will ionize hydrogen but optically thick for ionized helium radiation) for the energy balance temperature $\left(T_{\mathrm{EB}}\right)$ is given in Col. 4 of the table. For NGC 2022 Case I (completely optically thin) gives essentially the same temperature and for NGC 6818. Finally an average stellar temperature is given in Col. 5. These temperature estimates assume that the star radiates as a blackbody which, although probably not true, is a reasonable first approximation. This is indicated by the rather goed agreement of the various methods to determine the temperature. In addition, fitting a blackbody curve to the stellar continuum between $1400 \AA$ and $7000 \AA$ usually yields consistent temperature values (although this has never been done for the nebulae considered here).

\subsection{Radius and luminosity}

The stellar radius and luminosity are dependent on the distance of the nebulae which is difficult to obtain accurately. For this discussion we will use the distances cited above: $d=1.5 \mathrm{kpc}$ (NGC 2022), $d=2 \mathrm{kpc}$ (NGC 6818) and $d=2.1 \mathrm{kpc}$ (IC 4191). Similar values are cited in the literature especially as "individual distances". These values have an uncertainty which could be larger than $40 \%$. The distance, combined with the magnitude of the star, leads to the stellar radii which are shown in Table 22. Once the radius is known the gravity may be computed assuming that the star has a mass of 0.6 solar mass. The radius and the temperature give the luminosity shown in Table 22 .

It is also possible to obtain the stellar luminosity from the nebular $\mathrm{H} \beta$ luminosity, since there is a direct relationship between the number of ionizing photons and the number of $\mathrm{H} \beta$ photons, in the case in which the nebula absorbs all the ionizing photons emitted by the star. A mathematical formulation of this can be found in Pottasch \& Acker (1989). It yields the following luminosities: $L / L_{\odot}=150$ (NGC 2022), $L / L_{\odot}=1220$ (NGC 6818), and $L / L_{\odot}=765$ (IC 4191). These values maybe compared with the values given in Table 22. The large difference for NGC 2022 indicates that this nebula does not absorb all the ionizing photons, but allows a large number to escape from the nebula. On the other hand, IC 4191 and NGC 6818 appear to be optically thick to ionizing photons. The remaining differences being due to uncertainties in temperature (and the departures from blackbody radiation) and radius. This comparison does not give any information about the distance, since both formulations have the same distance dependance.
Table 22. Central star luminosities.

\begin{tabular}{lcccc}
\hline \hline Nebula & $\begin{array}{c}\text { Distance } \\
\mathrm{kpc}\end{array}$ & $\begin{array}{c}\text { Radius } \\
R / R_{\odot}\end{array}$ & $\begin{array}{c}\text { Gravity } \\
g / g_{\odot}\end{array}$ & $\begin{array}{c}\text { Luminosity } \\
L / L_{\odot}\end{array}$ \\
\hline NGC 2022 & 1.5 & 0.0655 & 140. & 852. \\
NGC 6818 & 2.0 & 0.0524 & 219. & 1090. \\
IC 4191 & 2.1 & 0.116 & 170. & 982. \\
\hline
\end{tabular}

\section{Discussion and conclusions}

The rather low luminosity of the central star of these nebulae, although quite uncertain, leads to the expectation that the initial mass of the star was low. In that case it is expected that only the first dredge-up (and possibly the third dredge-up) took place. The abundances which have been found seem to support this. Both NGC 2022 and NGC 6818 have abundances very similar to the sun, with the exception of carbon and sulfur. Sulfur appears to be a factor of 2 lower than the sun in all nebulae we have studied, which has also been found by Kwitter et al. (2003). We have earlier argued that the solar determination is at fault. The increased carbon abundance is undoubtably due to the third dredge-up. There is no indication of a large increase in either nitrogen or helium, indicating that no second dredge-up has occurred. As a consequence the central star masses must be less than about 3 solar masses, which is in agreement with their location rather far from the galactic plane.

The abundances in IC 4191 are a factor of from 1.5 to 2 higher than solar. Neon has an even higher overabundance. Even helium is $20 \%$ higher than solar. But because the nitrogen to oxygen ratio is almost solar, we do not feel that this increased abundance is an indication that the second dredgeup has taken place. It seems more likely that the star was born with these higher abundances. Judging from the carbon recombination line in this nebula, the carbon to oxygen ratio is very similar in this nebula to the ratio in the other two nebulae. It might appear that the higher abundance in this nebula is in contradiction to the conclusion that the initial mass of the star is low. There are however other effects which determine the abundance of the medium out of which the star is formed. One of the effects is the galactic position in which the star was formed. While this is difficult to ascertain, the fact that an abundance gradient based on the present galactic position has been found, is an indication that this might play an important role: IC 4191 is in the direction of the galactic center, while NGC 2022 is in the direction of the anti-center.

Acknowledgements. We would like to thank Dr. R. L. M. Corradi who gave many suggestions for improving the presentation of the paper.

Special notice. It is with sadness that we record the death of Walter Feibelman on 19 November 2004 at the age of 79, of a heart attack. It was quite sudden since I(S.R.P.) received an e-mail from him on 18 November in which he inquired about my health. I have known and worked with Walter for more than 30 years and his passing is a great loss to me. 


\section{References}

Acker, A., Marcout, J., Ochsenbein, F., et al. 1992, Strasbourg-ESO catalogue

Allende Prieto, C., Lambert, D. L., \& Asplund, M. 2001, ApJ, 556, L63

Allende Prieto, C., \& Prieto, D. L. 2002, ApJ, 573, L137

Aller, L. H., \& Czyzak, S. J. 1979, Ap\&SS, 62, 397

Aller, L. H., \& Czyzak, S. J. 1983, ApJS, 51, 211

Anders, E., \& Grevesse, N. 1989, Geochem. Cosmo., 53, 197

Becker, R. H., White, R. L., \& Edwards, A. L. 1991, ApJS, 75, 1

Benjamin, R. A., Skillman, E. D., \& Smits, D. P. 1999, ApJ, 514, 307

Bernard-Salas, J., Pottasch, S. R., Beintema, D. A., \& Wesselius, P. R. 2001, A\&A, 367, 949

Bernard Salas, J., Pottasch, S. R., Feibelman, W. A., \& Wesselius, P. R. 2002, A\&A, submitted

Cahn, J. H., Kaler, J. B., \& Stanghellini, L. 1992, A\&AS, 94, 399

Carrasco, L., Serrano, A., \& Costero, R. 1983, Rev. Mex. Astron. Astrofis., 8, 187

Ciardullo, R., Bond, H. E., Sipior, M. S., et al. 1999, AJ, 118, 488

Condon, J. J., \& Kaplan, D. L. 1998, ApJS, 117, 361

Corradi, R. L. M., Schonberner, D., Steffen, M., et al. 2003, MNRAS, 340,417

Davey, A. R., Storey, P. J., \& Kisielius, R. 2000, A\&AS, 142, 85

Feibelman, W. A. 1999, ApJ, 514, 296

Fluks, M. A., Plez, B., de Winter, D., et al. 1994, A\&AS, 105, 311

Gathier, R., \& Pottasch, S. R. 1988, A\&A, 197, 266

Gregory, P. C., \& Condon, J. J. 1991, ApJS, 75, 1011

Gregory, P. C., Vavasour, J. D., Scott, W. K., et al. 1994, ApJS, 90, 173

Grevesse, N., \& Sauval, A. J. 1998, Space Sci. Rev., 85, 161

Griffith, M. R., Wright, A. E., Burke, B. F., \& Ekers, R. D. 1994, ApJS, 90,179

Gutierrez-Moreno, A., \& Moreno, H. 1988, PASP, 100, 1497

Henry, R. B. C., Kwitter, K. B., \& Bates, J. A. 2000, ApJ, 531, 928

Hippelein, H., \& Munch, G. 1981, A\&A, 95, 100

Hummer, D. G., \& Storey, P. J. 1987, MNRAS, 224, 801

Hyung, S., Aller, L. H., \& Feibelman, W. A. 1999, ApJ, 514, 878

Keenan, F. P., Aller, L. H., Bell, K. L., et al. 1999, MNRAS, 304, 27

Keenan, F. P., Aller, L. H., Bell, K. L., et al. 1998, MNRAS, 295, 683
Kingsburgh, R. L., \& Barlow, M. J. 1994, MNRAS, 271, 257

Kwitter, K. B., Henry, R. B. C., \& Milingo, J. B. 2003, PASP, 115, 80 Liu, X.-W., Barlow, M. J., Cohen, M., et al. 2001, MNRAS, 323, 343

Liu, X.-W., Storey, P. J., Barlow, M. J., et al. 2000, MNRAS, 312, 585

Meaburn, J., Lopez, J. A., Bryce, M., \& Mellema, G. 1996, A\&A, 307, 579

Milne, D. K. 1979, A\&AS, 36, 27

Milne, D. K., \& Aller, L. H. 1982, A\&AS, 50, 209

de Freitas Pacheco, J. A., Maciel, W. J., Costa, R. D. D., \& Barbuy, B. 1991, A\&A, 250, 159

Pottasch, S. R., \& Acker, A. 1989, A\&A, 221, 123

Pottasch, S. R., \& Beintema, D. A. 1999, A\&A, 347, 974

Pottasch, S. R., Wesselius, P. R., Wu, C. C., et al. 1977, A\&A, 54, 435

Pottasch, S. R., Preite-Martinez, A., Olnon, F. M., et al. 1986, A\&A, 161,363

Pottasch, S. R., Beintema, D. A., \& Feibelman, W. A. 2000, A\&A, 363, 767

Pottasch, S. R., Beintema, D. A., Bernard Salas, J., \& Feibelman, W. A. 2001, A\&A, 380, 684

Pottasch, S. R., Beintema, D. A., Bernard Salas, J., et al. 2002, A\&A, 393, 285

Pottasch, S. R., Bernard Salas, J., Beintema, D. A., \& Feibelman, W. A. 2004, A\&A, 423, 593

Preite-Martinez, A., \& Pottasch, S. R. 1983, A\&A, 126, 31

Rowlands, N., Houck, J. R., Skrutskie, M. F., et al. 1993, PASP, 105, 1287

Schwarz, H. E., Corradi, R. L. M., \& Melnick, J. 1992, A\&AS, 96, 23

Shaw, R. A., \& Kaler, J. B. 1989, ApJS, 69, 495

Stanghellini, L., Corradi, R. L. M., \& Schwarz, H. E. 1993, A\&A, 276, 463

Taylor, A. R., Gussie, G. T., \& Goss, W. M. 1989, ApJ, 340, 932

Torres-Peimbert, S., Peimbert, M., \& Daltabuit, E. 1980, ApJ, 238, 133

Tsamis, Y. G., Barlow, M. J., Liu, X.-W., et al. 2003, MNRAS, 345, 186

Wang, W., Liu, X.-W., Zhang, Y., et al. 2004, A\&A

Wright, A. E., Griffith, M. R., Burke, B. F., \& Ekers, R. D. 1994, ApJS, 91,111 